\title{
ESTUDIO COMPARADO SOBRE TRANSPARENCIA Y DERECHO DE ACCESO EN EL ÁMBITO INTERNACIONAL Y SU INFLUENCIA EN ESPAÑA
}

COMPARATIVE STUDY ON TRANSPARENCY AND RIGHT OF ACCESS IN THE INTERNATIONAL SPHERE AND ITS INFLUENCE IN SPAIN

\section{Manuel Palomares Herrera*}

Resumen: Para comprender y asimilar la evolución actual y el alcance internacional de la transparencia pública y el derecho de acceso a la información pública holísticamente en nuestros días se propone un estudio de un conjunto internacional de normas de diferentes dimensiones. A tal efecto, sistémicamente, se lleva a cabo primeramente una compilación y clasificación de normas, declaraciones, disposiciones y resoluciones internacionalmente, y en segundo lugar a nivel europeo en las declaraciones comunitarias, las directivas y las resoluciones propias. A ello le sigue un comentario y un florilegio que trae a la luz la situación actual de la misma desde lo global a lo comunitario. El estudio concluye en una panorámica general de lo que se prevé es la dirección jurídica de un emergente derecho fundamental de acceso a la información pública, la tendencia y la evolución de la transparencia pública y su posterior impacto en el derecho comparado en el que se reflejan en los últimos acontecimientos en cada uno de los campos del derecho.

Palabras clave: Información pública, Unión Europea, normativa, Estado, jurisprudencia

* Doctorando en Derecho Público en la Universidad de Jaén, Andalucía (España). Abogado. Dirección: c/Paseo de la Estación 33ª 4º B, 23007, Jaén. Orcid Id: 0000-00031520-5036. manuel_cv_mph@outlook.es 
Abstract: In order to understand and assimilate the current evolution and the international scope of public transparency and the right of access to public information holistically today, a study of an international set of norms of different dimensions is proposed. To this end, systemically, a compilation and classification of international standards, declarations, provisions and resolutions is carried out first, and secondly a European level in Community declarations, directives and own resolutions. To this follows a commentary and a florilegio that brings to light the current situation of the same from the global to the community. The study concludes in a general overview of what lies in the direction of an emerging right fundamental right of access to public information, the trend and evolution of public transparency on comparative law in which they are reflected in the Recent developments in each field of law.

Keywords: Public Information, European Union, Legislation, State, Jurisprudence

Sumario. I. Proemio. II. Ámbito internacional. II.1. Introducción. II.2. Declaraciones internacionales. II.3. Disposiciones complementarias. II.4. Resoluciones de la Asamblea General de Naciones Unidas. III. Ámbito de la Unión Europea. III.1. Introducción. III.2. Declaraciones comunitarias. III.3. Directivas comunitarias. III.4. Otras disposiciones comunitarias aledañas. III.5. Jurisprudencia europea. III.6. Art. 255 del Tratado de Ámsterdam. IV. Conclusiones. Referencias.

\section{Proemio}

La irrupción mundial que en derechos y deberes sobre transparencia y acceso a la información paulatinamente se ha ido extendiendo desde Suecia, desde los países escandinavos, hasta asentarse con cierta homogeneidad en ambos hemisferios. Esta dinámica continúa extendiéndose por su influencia mutua, especialmente en Europa y América, así como en diversos organismos internacionales que se estudiarán en el presente artículo.

Cada país ha experimentado de distinta forma la implantación de Transparencias de Estado en función tanto del momento como del contendido. En casi todas las sociedades actuales la ciudadanía demanda convertirse en auténtico gestor de sus propios recursos, derechos y obligaciones, exigiendo a sus responsables público-políticos una rendición de cuentas respecto de sus actuaciones; para esto es necesario una información bidireccional de la actuación pública, como se observa en la 
mayor parte de países de la OCDE. En materia de transparencia - y sirva este apartado de motivación de peso para lo que se defiende - la normativa internacional ha vivido importantes cambios debido a la irrupción que han tenido las nuevas tecnologías en lo que se refiere al acceso a la información, que hace que se cambie la forma de gestionar el servicio público, que se elaboren e implementen las políticas públicas como estas, y todo ello tiene como resultado una mejora de la calidad democrática. Guichot afirmaba que «el derecho de acceso no constituye una pieza única para una sociedad transparente, pero sí resulta pieza fundamental para lograrlo» (2014, p. 14).

Por ello, desde que en Suecia, actual Estado de transparencia, se publicase la Ley de Acceso a la Información en el año 1766, que fue la primera normativa de transparencia de Estado directa, otros muchos países del mundo consideraron que era importante incluir y perfeccionar tal normativa en sus ordenamientos jurídicos. Aunque no se estudiarán todos los países en esta obra por cuestión de espacio, veremos casos especiales como es el evolucionado caso de Sudáfrica con su ley de 2000, la cual difiere del resto porque obliga a publicar la información hasta a las empresas privadas, cuando se considerare que la información es relevante para cualquier derecho fundamental, como por ejemplo el acceso al agua.

$\mathrm{Si}$ a finales de siglo $\mathrm{XX}$ eran solo trece los países los que disponían de una Ley de este estilo, ahora son más de ochenta y cerca de veinte están en proceso de su tramitación y aprobación. Pese a ello, ha de mencionarse a países que muestran la otra cara de la moneda como son los países moros o como India que, aunque cuenta desde 2005 con una ley con mecanismos muy detallados para garantizar la transparencia, en la práctica muestra otra realidad porque muchas personas han sido asesinadas por defender esta ley.

Esta tendencia global de mainstreaming, de aprobación de normas garantes del acceso a la información pública, comenzó en los países escandinavos. Empezó en la indicada Suecia en 1766, luego pasó a Finlandia en 1951, a Estados Unidos en 1966, a Noruega en 1970 y a otros países de influencia anglófona como Canadá, Nueva Zelanda y Austria. Más tarde se expandió entre los países del mediterráneo de la Europa occidental, como Francia en 1978, Grecia en 1986, Italia en 1990, Portugal en 1993 o Bélgica al año siguiente. La segunda oleada alcanzó en el nuevo siglo a los Estados de Europa occidental que aún carecían de esta Ley, como el Reino Unido en el año 2000 y Alemania cinco años después.

En el caso particular de España, algunas de las reglas de transparencia que se alojan en las Leyes de Transparencia internacionales quedaron en preceptos de Transparencia de Estado indirecta de otros cuerpos normativos como los artículos 3.5, 35 y 37 de la Ley 30/1992 de 
Régimen Jurídico de la Administración (RJPAC), antes de la publicación de la Transparencia de Estado directa española por antonomasia, la Ley de Transparencia, Acceso a la Información y Buen Gobierno 19/2013 (LTBG).

Por ofrecer un prisma determinado a nuestro ordenamiento respecto a transparencia y acceso a la información, se ha de observar la regulación que hacen los Estados vecinos a España en la Unión Europea (UE) por el entorno jurídico comunitario que nos afecta, así como a los Estados latinoamericanos por las raíces históricas que nos han unido y que tanto nos influyen. En el estudio excluimos la experiencia de Estados que pese a su volumen geo-político y comercial (China, Rusia y EE.UU.) han aportado irrelevantes elementos en la materia que nos ocupa.

En definitiva, la dimensión extrafronteriza de derechos de acceso y transparencia solo puede darse a la luz por medio de la herramienta jurídica del Derecho comparado. Buscaremos distinguir las diversas soluciones que ofrecen los distintos ordenamientos jurídicos para los mismos casos. Dado que ciencias como la jurisprudencia a modo de pseudo-fuente del derecho acuden ya al Derecho comparado para interpretar las normas jurídicas, se ve conveniente hacer un apunte general para motivar y para ver por dónde y por qué puede evolucionar el ordenamiento español, y en qué medida puede el nuestro influir en los demás.

Este estudio comparado se ampara en la defensa del reconocimiento del derecho de acceso a la información pública como derecho fundamental en el ordenamiento jurídico español - donde aún no existe normativa específica sobre el tema-, tanto por razones de índole jerárquiconormativa como de propia lógica de concepción mundial de este derecho.

\section{II. ÁMBITO INTERNACIONAL ${ }^{1}$}

\section{II.1. Introducción}

Es en el ámbito internacional donde organismos de calado internacional para cada uno de los Estados, sean o no vinculantes para todos, definen, recomiendan, tasan y suscriben normas de acceso a la información y de transparencia y otras transparencias de Estado que estudiaremos y comentaremos. El que España haga vaga fe de cumplimiento de estas exigencias, amparándose en el art. 105. b) de la Constitución Española (CE) impide que haga gala como otros Estados de la presencia de estas evocaciones en los espacios constitucionales donde figuran los derechos fundamentales. Como se entiende por extractos y

\footnotetext{
${ }^{1}$ Cfr. Diez De Velasco, 2009.
} 
comentarios, es un derecho humano de nuevo cuño y generación el derecho de acceso y que cada vez aleja más nuestra CE de estas nuevas miras a las que, incumpliendo o desobedeciendo, no se logrará más que ampliar la distancia que separa a nuestro Estado de Derecho de alcanzar el Estado de la transparencia.

\section{II.2. Declaraciones internacionales}

Inmiscuidos en lo que Diez De Velasco llama «proceso de internacionalización de los Derechos Humanos» ${ }^{2}$ y en referencia al derecho de acceso y transparencia, se ha de considerar la Declaración de los Derechos del Hombre y del Ciudadano de 1789 como el germen de las declaraciones mundiales. Ahí encontramos notorias referencias a la transparencia, como estas:

Art. 14. Los ciudadanos tienen el derecho de comprobar, por sí mismos o a través de sus representantes, la necesidad de la contribución pública, de aceptarla libremente, de vigilar su empleo y de determinar su prorrata, su base, su recaudación y su duración.

Art. 15. La sociedad tiene derecho a pedir cuentas de su gestión a todo agente público.

El primer precepto ensalza el poder ciudadano respecto a sus gestores, mientras el segundo reconoce ampliamente el derecho a exigir a la administración la rendición de cuentas de los recursos públicos.

Más tarde, en un marco postguerras, aparece la Declaración Universal de Derechos Humanos de 1948 donde la transparencia sigue siendo un objetivo en continuo desarrollo y evolución. Así se deduce de su art. 19, que señala que «Todo individuo tiene derecho a la libertad de opinión y de expresión; este derecho incluye el de no ser molestado a causa de sus opiniones, el de investigar y recibir informaciones (...)».

Es llamativa la síntesis que el legislador universal utiliza al reunir en un mismo apartado tal cantidad de principios y valores, que por su vinculación ha decidido conectar. Guichot (2014, p. 35) ya hablaba de la igualdad como límite y como derecho en el libre acceso a la información, pero este texto va más allá al crear la accesoriedad del derecho de acceso a

${ }^{2} \mathrm{Al}$ respecto, Diez De Velasco (2009, pp. 648-650) señala:

«A partir de la segunda mitad del siglo XX un conjunto de normas dedicadas a la protección internacional del individuo se agrupan bajo la categoría genérica de Derecho Internacional de Derechos Humanos (...) el resultado ha sido la adopción de normas sustantivas que enuncian derechos, algunas de las cuales han llegado a integrarse en el bloque cualificado de las normas de ius cogens». 
la información al ejercicio del derecho de libertad de expresión, la cual se ha de respetar cuando se solicite o investigue cierta cuestión pública legítima. La mayor perturbación de la recepción de la información que expone este precepto es la inexistencia de un procedimiento que faculte a solicitar una información, para lo que se requiere el reconocimiento máximo del derecho de acceso.

Un componente básico de la Carta Internacional de Derechos Humanos - eximia declaración internacional también en transparencia y acceso a la información - es el Pacto Internacional de Derechos Civiles y Políticos. Es este pacto adoptado el 16 de diciembre de 1966 acordó en materia de transparencia que «nadie podrá ser molestado a causa de sus opiniones» y que «toda persona tiene derecho a la libertad de expresión» (art. 19, nums. 1 y 2), derechos que comprendían otras libertades.

Recuerda su apartado segundo al artículo descrito de la Declaración Universal de Derechos Humanos por ser prácticamente igual. La diferencia radica en su desarrollo que encuentra en el punto 3, donde por primera vez aparece a nivel internacional un amago de concebir la transparencia activa (el deber de las Administraciones Públicas de ofrecer información) y la previsión de unos límites al derecho de acceso a la información —a los que denominó de "restricciones" - por razones de orden público, moralidad o la de seguridad nacional (lo último está presente en la LTBG).

Desde una óptica moderna y desde el sur occidental del mapamundi se vuelven a declarar internacionalmente las premisas humanistas de la transparencia y el derecho de acceso por medio de la Convención Americana sobre Derechos Humanos en el año 1969, en San José de Costa Rica. El sector sur-continental americano firmó su Convenio en el que en cinco pragmáticos puntos de un mismo precepto titulado "Libertad de Pensamiento y Expresión" se reconoció la transparencia. El precepto reza así: «Toda persona tiene derecho a la libertad de pensamiento y de expresión. Este derecho comprende la libertad de buscar, recibir y difundir informaciones e ideas de toda índole (...)» (art. 13).

Como en el anterior caso, se limitan al honor, seguridad nacional y moral las líneas rojas de la recepción y emisión de información, añadiéndose las razones de salud. Pese a que hasta ahora no se hace mención expresa al carácter público de la información, así se ha interpretado por jurisprudencia que a continuación se tratará. Resulta llamativa también la inclusión de la censura, puesto que a mediados del siglo XX ya gozaba esta de un rechazo considerable por ser un filtro gubernamental usado internacionalmente con más fines políticos que estatales. Se podría decir que todas las manifestaciones expuestas hasta el momento están presentes en el primer título de nuestra Constitución, a 
excepción de las concretas expresiones «recepción de informaciones», que propiamente no es una exigencia tal como se concibe actualmente.

Es de justicia señalar un modelo y precedente pionero en su época, que antes de la declaración en cuestión ya tomaba en consideración el derecho de acceso por medio del derecho de petición. Nos referimos a la Declaración Americana de los Derechos y Deberes del Hombre de 1948, aprobada en la $9^{\text {a }}$ Conferencia Internacional Americana celebrada en Bogotá.

A pesar de ser una declaración restrictiva a reconocer los derechos más inmediatos, el derecho a solicitar informaciones de interés general está presente en su art. 24 que dice así: «Toda persona tiene derecho de presentar peticiones respetuosas a cualquiera autoridad competente, ya sea por motivo de interés general, ya de interés particular, y el de obtener pronta resolución».

Terminamos con una afirmación tajante de la sensible Declaración Interamericana de Principios sobre la Libertad de Expresión del año 2000, donde - en un adelanto importante- se aprobó lo que es el documento oficial más completo hasta la fecha sobre la libertad de expresión en el sistema interamericano (Mendel, 2008, p. 19). El Preámbulo reafirma las declaraciones mencionadas sobre el derecho a la información:

«El acceso a la información en poder del Estado es un derecho fundamental de los individuos. Los Estados están obligados a garantizar el ejercicio de este derecho. Este principio sólo admite limitaciones excepcionales que deben estar establecidas previamente por la ley para el caso que exista un peligro real e inminente que amenace la seguridad nacional en sociedades democráticas».

\section{II.3. Disposiciones complementarias}

Fruto de la implicación internacional en la materia se declararon en una escala menor a la de las declaraciones anteriores unas proposiciones legislativas, interesantes por su ambición y por haber desarrollado preceptos y elementos que manifiestan la transparencia y el derecho de acceso como altos valores, como sucedió en la Carta Democrática Interamericana.

En esta recopilación se encuentra un instrumento que proclama como objetivo principal el fortalecimiento y preservación de la institucionalidad democrática estableciendo que la ruptura del orden democrático o la modificación que afecte gravemente el orden democrático en un Estado miembro, constituirá «un obstáculo insuperable» para la participación de su gobierno en las diversas instancias de la OEA. Esto manifiesta la subordinación que la administración ha de sufrir respecto a la ciudadanía a la que sirve, razón de su existencia. Extrapolándolo al caso español, la 
subordinación y respeto de lo administrativo a lo dogmático solo se puede concebir desde la concesión del rango fundamental al derecho de acceso y de ley orgánica a la LTBG.

La Carta Democrática Interamericana de 2001, implicó además el compromiso de los Estados con la democracia, basado en el reconocimiento de la dignidad humana. Su art. 4 señala que «son componentes fundamentales del ejercicio de la democracia la transparencia de las actividades gubernamentales, la probidad, la responsabilidad de los gobiernos en la gestión pública, el respeto por los derechos sociales y la libertad de expresión y de prensa (...)».

Siguiendo a la anterior Carta, ha de destacarse el texto al que España adhirió y aprobó recientemente, la Carta Iberoamericana de Participación Ciudadana en la Gestión Pública ${ }^{3}$.

Es por este desarrollo normativo por el que, tanto por razones de extensión como por razones de concentración del estudio en el derecho de acceso y transparencia de Estado, se hará referencia focalizadamente a su parte dogmática y a las secciones de su parte orgánica que brindan la oportunidad comparativa de estudiar la transparencia de Estado dentro de la participación ciudadana.

Según su preámbulo inicial, se considera que una gestión pública es buena si cumple satisfactoriamente con una serie de parámetros preestablecidos de integridad, eficiencia, eficacia, efectividad, igualdad y equidad, referenciados a un impacto positivo sobre el desarrollo social y la calidad de vida de las personas, para lo cual debe ser esencialmente democrática, lo que garantiza el logro de niveles de calidad y excelencia en su desempeño y resultados.

Según el texto, «el derecho de participación ciudadana en la gestión pública conlleva establecer mecanismos para ello, complementarios a los previstos para la representación política en el Estado. La gestión pública participativa contribuye al desarrollo de los países (...)», la misma que se desprende del «derecho de participación ciudadana en la gestión pública». Este derecho corresponde a todo habitante iberoamericano, con respecto a la gestión pública del país en que reside en el ejercicio de los derechos que le conciernen o, en su caso, a la gestión pública vinculada a los procesos de integración regional o subregional. Así, el título de "ciudadano" y "ciudadana" en la Carta Iberoamericana no está referido a las personas con derechos exclusivos de ciudadanía o de nacionalidad, sino a todo habitante

\footnotetext{
${ }^{3}$ En la XI Conferencia Iberoamericana de Ministros de Administración Pública y Reforma del Estado en Lisboa (Portugal) el 25 y 26 de junio de 2009, texto que cuenta con 50 arts.
} 
con respecto a la gestión pública del país en donde reside, en el ejercicio de los derechos que le conciernen.

El mayor desafío de la participación ciudadana, en la gestión pública en el año 2009 era impulsar su universalización para crear unas condiciones que permitan que los sectores más vulnerables pudieran acceder a la participación ciudadana para la defensa y exigencia de sus propios derechos, estableciéndose esta facultad como un medio novedoso y de calidad para la transformación social.

En el capítulo tercero, como forma de participación en la gestión, se cita en su art. 22 la capacidad o aptitud del ciudadano de solicitar información pública para garantizar la participación individual y colectiva en las administraciones públicas. Concretamente dice:

Art. 22. a) El acceso a información de interés general, su difusión activa y la posibilidad de consulta a través de medios físicos, audiovisuales y electrónicos. (...)

g) La gestión transparente de intereses a instancias de colectivos ciudadanos para manifestar reclamos, propuestas o consultas en el marco de las políticas y normas vigentes.

Excelente ejercicio de síntesis en dos pautas que se complementan. Es posible, recomendable y a futuro, necesario, el reconocimiento máximo en las cartas magnas de los Estados, el acceso a la información y el deber transparencia. Así es como los Estados iberoamericanos consagraron el derecho a reclamar derechos a través de acciones ciudadanas de interés público, entre las que figuran las piezas que se estudian.

El capítulo $5^{\circ}$ ilustra el grueso del derecho de acceso rotulándolo "Acceso a la información pública para la participación ciudadana", concibiendo de esta manera el derecho de acceso a la información como sustento de la participación en los dos siguientes numerales:

Art. 40. El acceso a la información es un derecho que sustenta el adecuado funcionamiento de la democracia puesto que es condición para garantizar otros derechos y, en particular, el de participación ciudadana en la gestión pública (...).

Art. 41. Para hacer efectivo el derecho de acceso a la información pública, los ciudadanos (...) podrán (...):

a. Acceder a la información que obre en poder de las Administraciones Públicas, sin que para ello estén obligados a declarar un interés determinado;

b. Acceder a los archivos y registros de las Administraciones Públicas;

c. Ser asistidos en su búsqueda de información;

d. Recibir la información que soliciten, en los plazos máximos establecidos en la norma correspondiente;

e. Recibir la información solicitada en forma o formato accesible para quienes la demanden (...). 
Sin menospreciar las amplias observaciones derivadas del derecho de acceso, particularmente llama la atención en la técnica redactora el forzado e indecoroso uso de desdoblamientos sexistas como "ciudadanos y ciudadanas", tan de tendencia igualitaria en la época, aunque la Real Academia de la Lengua Española señala que no es correcto, ni por economía del lenguaje, ni por adecuación gramatical.

La transparencia en la gestión pública se califica y corona como principio algo que hasta el momento solo figuraba en los estudios de esta rama como aspiración, pero que en reducidos ordenamientos así se engendró:

Art. 42. La transparencia es un principio que debe orientar la relación de la Administración con los ciudadanos y las ciudadanas en el manejo de la gestión pública.

Art. 43. Los Estados adoptarán las medidas adecuadas para que la información puesta a disposición de los ciudadanos y las ciudadanas garantice el principio de transparencia (...).

A pesar de las críticas que la LTBG reciba en su apartado correspondiente, ha de parcialmente reconocerse su cercanía a las líneas sugeridas en la Carta. No olvida tampoco esta Carta la transparencia activa, en la que se ha mirado la LTBG por cumplir los parámetros más básicos a los que se refiere de la siguiente forma:

Art. 44. El Estado pondrá a disposición, sin que necesariamente medie solicitud de los ciudadanos y las ciudadanas, información actualizada (...).

Art. 45. Las Administraciones Públicas pondrán a disposición de los ciudadanos y ciudadanas la información relativa a los servicios y prestaciones que ofrecen y los requisitos, condiciones, trámites y plazos de los procedimientos administrativos y los medios para acceder a ellos.

Art. 46. Los entes y órganos públicos dispondrán de sistemas de organización (...) para facilitar la participación ciudadana.

En el marco de la OCDE se encuentra otra disposición internacional relativa al estudio, el Convenio OCDE de Convenio de lucha contra la corrupción de agentes públicos extranjeros en las transacciones comerciales internacionales de 1997, que estuvo enfocado a los delitos por extracción de beneficios salientes de arcas públicas valiéndose de un cargo público. Este Convenio, ha sido suscrito hasta la fecha por 41 países: los 34 miembros de la OCDE, más Argentina, Brasil, Bulgaria, Colombia, Letonia, Rusia y Sudáfrica.

El Convenio tiene entre sus objetivos el de garantizar una competencia libre de vicios, libre de prácticas corruptas. Este objetivo se 
ha traducido en el compromiso de adoptar una serie de modificaciones legales que se estimen oportunas y convenientes para poder reprimir tales actos, como los actos de soborno a funcionarios públicos extranjeros realizados por sus nacionales con la intención de conseguir o conservar un contrato u obtener otro beneficio irregular en la realización de actividades económicas internacionales. El Convenio abarca cuatro ámbitos:

(i) Penal: se establece la obligación de definir como delito y castigar el cohecho realizado para la obtención de algún contrato internacional. Este instrumento también sanciona la complicidad.

(ii) Contable: se hacen recomendaciones a las empresas para prevenir el encubrimiento de algunos gastos en los registros contables. Se sancionan las omisiones, alteraciones, fraude y falsificaciones de registros, cuentas, estados financieros y libros con el propósito de ocultar el cohecho.

(iii) Blanqueo de dinero: el hecho de detectar un pago de soborno ilegal relacionado con transacciones internacionales es suficiente para provocar la aplicación de la legislación contra el blanqueo de capitales para que esos pagos puedan ser confiscados.

(iv) Asistencia legal mutua: si se utilizan canales financieros internacionales para ocultar o realizar el cohecho internacional, el Convenio obliga a los países signatarios a proveer el apoyo legal oportuno, además de establecer provisiones relativas a la extradición.

\section{II.4. Resoluciones de la Asamblea General de Naciones Unidas ${ }^{4}$}

Uno de los dictámenes internacionales a valorar es la Resolución de la Asamblea General sobre Acceso a la información pública y protección de datos personales AG/RES. 2661 (XLI-O/11). Se trata del primer texto supranacional firmado con un reconocimiento directo y exclusivo al derecho de acceso. Pese a que la Organización de Estados Americanos no tiene influencia, ni vinculación alguna con nuestro ordenamiento jurídico, un estudio de derecho comparado debe ver estos reconocimientos de derechos de organismos supranacionales homólogos a la UE. Este ente, amparándose en anteriores resoluciones del mismo órgano como la de "Acceso a la información pública: Fortalecimiento de la democracia", la "Ley Modelo Interamericana sobre Acceso a la Información Pública" y reiterando todos los mandatos y consideraciones históricas, procedimentales, jurídicas, políticas y sustantivas contenidas en éstas, resuelve en la $4^{\text {a }}$ sesión plenaria el 7 de junio de 2011, lo siguiente:

\footnotetext{
${ }^{4}$ Para ahondar en la repercusión y naturaleza de estos dictámenes, cfr. Castañeda, 1967.
} 
«1. Reafirmar la importancia del acceso a la información pública como requisito indispensable para la democracia y el compromiso de los Estados Miembros de respetar y hacer respetar el principio de brindar acceso a la información gubernamental.

2. Alentar a los Estados a que en el diseño, ejecución y evaluación de sus normativas y políticas sobre acceso a la información pública, consideren la aplicación e implementación de la Ley Modelo Interamericana sobre Acceso a la Información Pública contenida en la resolución AG/RES. 2607 (XL-O/10) y su Guía de Implementación.

3. Encomendar a la Secretaría General, a través del Departamento de Derecho Internacional, que con la colaboración de la sociedad civil apoyen los esfuerzos de los Estados miembros de tomar las medidas (...)».

En primer lugar comienza la resolución con una definición, delimitando el acceso a la información pública, por un lado, y la protección de datos personales, por el otro, como valores fundamentales que deben trabajar siempre en concordancia. De los once puntos, los cuatro primeros nos interesan especialmente en este estudio, siendo el resto meras formalidades protocolarias fruto de su ejercicio administrativo en relación a una serie de comisiones internas y análogas.

De los primeros apartados se extrae una primordial vinculación del derecho de acceso a la información con el buen gobierno y con las democracias. No en todos los Estados Americanos se respetan estas garantías. Piénsese en Venezuela, Haití, Honduras y similares, que ni aparentan el respeto a los derechos humanos. Muchos no aspiran a un Estado de transparencia, porque no han pertenecido permanentemente a esta organización por razón de sus gobiernos y situación política, o porque a pesar de pertenecer no gozan del aparato económico-político de impulso de garantías sociales de transparencia de Estado.

En esta resolución se menciona la figura del Comisionado $u$ Ombudsman ${ }^{5}$ como sujeto orgánico sugerido a responsabilizarse de la garantía del derecho de acceso en cada Estado. Si bien en España el Defensor del Pueblo tiene funciones contencioso-administrativas, según se extrae de la LTBG, y es un auxilio alternativo en jurisdicciones como sujeto de acción judicial y vía constitucional de amparo, es el CTBG el que asume las premisas en transparencia, al igual que en la mayoría de países.

5 Existen figuras análogas en el resto del mundo, como el Mediateur Français, el Ombudsman de Noruega o Suecia o el Comisario Parlamentario Británico; en España es denominado Defensor del Pueblo y muestra una variedad de formas de implantación, con las mismas funciones, como el denominado Valedor de Pobo Gallego, Justicia Mayor de Aragón, Procurador Común de Castilla, Ararteko Vasco, Síndic de Greuges en Cataluña, el Diputado del Común Canario o el Defensor del Pueblo Andaluz. 
Luego, debemos aludir a la Resolución de la Asamblea General sobre la Ley Modelo Interamericana de Acceso a la Información Pública AG/RES. 2607 (XL-O/10), previa a la antes estudiada. Ella introduce y reafirma ${ }^{6}$ la Ley Modelo Interamericana sobre Acceso a la Información Pública de 2010, la cual resolvió que «el acceso a la información es un derecho humano fundamental y una condición esencial para todas las sociedades democráticas» y que «se aplica en sentido amplio a toda la información en posesión de órganos públicos, incluyendo toda la información controlada y archivada en cualquier formato o medio». Aquí recoge una gran catalogación de este derecho, su carácter de humano y fundamental, y con este reconocimiento - asimilable a un estatuto o ley marco - se establece el espejo en que los Estados del continente han de mirarse.

Tras la primera Ley Marco de Transparencia y Acceso a la Información supranacional del mundo, la OEA implanta la Ley Modelo, con las siguientes premisas:

«El derecho de acceso a la información está basado en el principio de máxima divulgación de la información;

Que las excepciones al derecho de acceso a la información deberán ser clara y específicamente establecidas por la ley;

Que aun en la ausencia de una petición específica, los órganos públicos deberán divulgar información sobre sus funciones de forma regular y proactiva, de manera que asegure que la información sea accesible y comprensible (...)».

Hoy se podría ver esta resolución con un carácter sucinto o primitivo, dado el extraordinario desarrollo que ha experimentado la materia en los últimos lustros, pero ha de reconocerse el mérito democrático de incluir los mencionados elementos. Se trata de una resolución adelantada a su época y a nivel internacional fue precursora de las normas que iban a implantarse.

En conclusión, en pocas líneas se establecen los elementos generales de un procedimiento con garantías para solicitar información que hoy ya está aceptado, pero que entonces supuso un avance en el momento en el que se propuso el aval de instancias superiores en caso de negativa, así como garantizar la integridad del mismo, la seguridad jurídica de la petición, las dinámicas de buen gobierno a través de disuasiones por medio de sanciones, obligando a la gratuidad de la solicitud u oferta de información top down o de transparencia activa.

${ }^{6}$ En sus dos puntos primeros indica:

1. Tomar nota de la Ley Modelo Interamericana sobre Acceso a la Información, documento CP/CAJP-2840/10, que forma parte de esta resolución, así como de la guía para su implementación, contenida en el documento CP/CAJP-2841/10. 2. Reafirmar, en lo que resulte aplicable, los mandatos contenidos en la resolución 


\section{III. ÁMBITO DE LA UNIÓN EUROPEA}

\section{III.1. Introducción ${ }^{7}$}

Desde el origen de la UE a mediados de siglo XX, en plena formulación de las líneas que preparaban los primeros pactos económicos (CECA, Euratom, etc.), encontramos en los Tratados constitutivos manifestaciones relativas a la transparencia de las instituciones comunitarias. Conforme avanzaba la consolidación de la misma, las disposiciones sobre transparencia fueron aplicándose a otras materias como medio ambiente, prensa o igualdad.

Comenzamos señalando la Declaración $\mathrm{n}^{\mathrm{o}} 17$ adjunta al Acta final del Tratado de la $\mathrm{UE}^{8}$ firmado en Maastricht, como sólido punto de partida en esta materia. Aquí se reconoce la primacía del Derecho europeo sobre el individual de los Estados, con el objetivo de obtener la confianza de las personas en las instituciones jurídicas europeas, con lo que el trasvase de derechos desde este momento ha sido incesante.

Con la celebración de los sucesivos Consejos Europeos se sentaron las bases de la política sobre transparencia comunitaria. Poco a poco las instituciones alcanzaron acuerdos favorables a la transparencia aprobando los Códigos de acceso a los documentos del Consejo y de la Comisión, cuyos principios inspiraron más tarde las normas vinculantes en dicha materia, que adoptaron otras instituciones, órganos y organismos comunitarios. Estos Códigos de Buena Conducta son los que establecieron, entre otros, los principios a cumplir por los funcionarios comunitarios en los procesos de solicitud de acceso a documentos obrantes en el seno orgánico de la UE. Esto supuso un avance adicional en sistemas informáticos o internet, que facilitó acceder a la información pública europea mediante cualquier ordenador.

Ha de destacarse también la labor del Defensor del Pueblo como institución europea en esta materia en la promoción de adopción de medidas de transparencia del Estado, sobre acceso a la información pública, mediante la investigación y emisión de informes.

Con el Tratado de Ámsterdam de 1997 se inaugura una nueva etapa en materia de transparencia comunitaria. Se introduce el principio de

${ }^{7}$ Cfr. Álvarez \& Alcón, 1996; Cotino Hueso, 2005.

${ }^{8}$ Esta Declaración relativa a la primacía rezaba:

«La Conferencia recuerda que, con arreglo a una jurisprudencia reiterada del Tribunal de Justicia de la Unión Europea, los Tratados y el Derecho adoptado por la Unión sobre la base de los mismos priman sobre el Derecho de los Estados miembros, en las condiciones establecidas por la citada jurisprudencia». 
apertura en la toma de decisiones, se declara el derecho de acceso a los documentos del Parlamento Europeo, del Consejo y de la Comisión, y se establecen los medios de impugnación frente a resoluciones denegatorias de solicitudes de acceso.

Seguidamente el Tratado Constitucional de 2003 incluyó los principios de democracia representativa y participativa, al tiempo que extendió el derecho de acceso a los documentos ya que al no entrar totalmente en vigor este, todos los principios en él contenidos, fueron transferidos al Tratado de Lisboa (Quirós, 2012, p. 193).

Los dos buques insignias de transparencia de éxito, de exposición y de limpieza de la UE son el famoso Portal de Transparencia Europeo y el Registro de transparencia de la UE. Otro de los logros comunitarios para todos los que gozan de ciudadanía europea fue el derecho a saber cómo se cuajan las decisiones institucionales europeas, quiénes participan en su elaboración, quién y en concepto de qué recibe financiación del presupuesto de la UE, y en qué documentos se basan la preparación y la adopción de los actos normativos europeos, junto con el derecho a acceder a esos documentos y a manifestar su opinión, ya sea directa o indirectamente, a través de los intermediarios que les representan.

Estas piezas fueron los referentes sobre la materia para los Estados miembros, junto con los elementos que van a desarrollarse.

\section{III.2. Declaraciones comunitarias}

Primero está el Convenio Europeo para la Protección de los Derechos Humanos y las Libertades Fundamentales, firmado en Roma el 4 de noviembre de 1950. Se trata de la primera declaración europea que reconoce un derecho a "recibir información"9. Su art. 10.1 consagra la libertad de expresión en los siguientes términos: «toda persona tiene derecho a la libertad de expresión. Este derecho comprende la libertad de opinión y la libertad de recibir o de comunicar informaciones (...)».

La próxima alusión de renombre en términos de acceso a la información no aparecerá sino hasta finales de siglo XX con el Convenio sobre acceso a la información, participación del público en la toma de decisiones y acceso a la justicia en materia medioambiental de 1998. Fue firmada en Dinamarca y se conocía normalmente como "Convenio de Aarhus", al que puede definirse como tratado internacional que regula los

\footnotetext{
${ }^{9}$ Reconocido y firmado por los 47 Estados pertenecientes al Consejo de Europa, forma este convenio parte de la Carta Europea de Derechos Humanos junto a un conjunto de protocolos y pactos.
} 
derechos de participación ciudadana en relación con el medio ambiente en el marco de la antigua Comunidad Económica Europea.

El acceso a la información en materia medioambiental es el primero de los derechos regulados por el Convenio de Aarhus y constituye un requisito previo para que puedan ejercerse el resto de derechos regulados en el mismo. El tratado recoge el derecho de acceso a la información tanto en su vertiente activa en su art. 5, como en su vertiente pasiva en su art. 4. El art. 5 señala la obligación de las administraciones públicas de difundir la información ambiental que poseen, y concretamente los informes sobre la situación del medio ambiente en cada Estado, al menos cada cuatro años. La vertiente pasiva, en cambio, implica la obligación de entregar a los ciudadanos la información en materia de medio ambiente que soliciten, en el plazo máximo de un mes.

La Carta de los Derechos Fundamentales de la Unión Europa fue el primer cuerpo jurídico del milenio en evocar, instaurar y elevar a dicho rango el derecho de acceso a los documentos de carácter público. Adoptada en Niza el año 2000, contiene los derechos de recepción de información, protección y acceso a datos, archivos y expedientes de carácter personal, a la buena administración, obligación de la administración, la obligación de motivar sus decisiones y el acceso a los documentos de las instituciones europeas según los siguientes artículos:

Art. 11.1. Toda persona tiene derecho a la libertad de expresión. Este derecho comprende la libertad de opinión y la libertad de recibir o de comunicar informaciones o ideas sin que pueda haber injerencia de autoridades públicas y sin consideración de fronteras (...).

Art. 42. Todo ciudadano de la Unión o toda persona física o jurídica que resida o tenga su domicilio social en un Estado miembro tiene derecho a acceder a los documentos del Parlamento Europeo, del Consejo y de la Comisión.

Casi una década después, el Convenio del Consejo de Europa sobre el Acceso a los Documentos Públicos firmado en Troms, Noruega el 18 de junio de 2009, fija un derecho de acceso a los documentos públicos en la que cada parte

«garantizará el derecho de cualquiera, sin discriminación de ningún tipo a acceder, bajo petición, a los documentos públicos en posesión de las autoridades públicas. 2) Cada Parte tomará las medidas necesarias en su ordenamiento jurídico para hacer cumplir las previsiones sobre acceso a documentos públicos previstas en este Convenio. 3) Estas medidas deberán ser adoptadas por cada Parte a más tardar en el momento de la entrada en vigor de este Convenio» (art. 2).

Por su parte, el art. 6.1 establece que a la firma del Convenio los Estados firmantes habrán de establecer unas formas concretas de acceso: 
«cuando se concede el acceso a un documento oficial, el solicitante tiene el derecho a elegir si examina el original o una copia, o si recibe una copia en cualquier forma disponible o en el formato que elija (...)».

\section{III.3. Directivas comunitarias}

Como Fernández Ramos (1997) describe, eran varias las disposiciones relativas a la transparencia administrativa en el Tratado de la Comunidad Económica Europea ${ }^{10}$, si bien no hubo un deber taxativo que plasme la publicidad activa en el ámbito administrativo comunitario previo al Tratado de la UE, hasta que el 23 de enero de 1988 el Parlamento de la UE aprobase la resolución de publicidad de procedimientos comunitarios en la que se estima el derecho a la información como un derecho fundamental del ciudadano europeo. Esta es la Directiva 90/313/CEE.

La primera directiva en este campo, que ha evolucionado mucho a lo largo del tiempo, está dedicada solo a la información pública medioambiental. Recuerda Fernández Ramos (1997, p. 234) que este interés nace en la Resolución de 19 de octubre de 1987, relativo al cuarto programa de medio ambiente de la Comunidad Europea (1987-1992), donde el Consejo de los Estados miembros, reunidos en el seno del Consejo declararon la necesidad de mejorar el acceso a la información sobre el medio ambiente. El propio programa recomendó la elaboración de procedimientos para que el público tenga mayor acceso a la información que las autoridades responsables del medio ambiente tienen en su poder.

Otro desarrollo interesante nace en el preámbulo de la propuesta de Directiva del Consejo sobre libertad de información en materia de medio ambiente 88/C 335/04, presentada por la Comisión el 31 de octubre de $1988^{11}$, que afirma la «necesidad de mayor transparencia en todo el proceso de reglamentación y aplicación de las normas existentes, en particular en todo lo relativo a la información pública». Su primer precepto «garantiza en toda la Comunidad Europea la libertad de acceso a la información especialmente la medioambiental en poder de la autoridad

${ }^{10}$ El único antecedente que ofrece Fernández Ramos como ejemplo de la escasez de materia normativa en acceso a información es el siguiente:

«(C)on el triple objeto de fomentar la investigación sobre la historia del funcionamiento de las instituciones europeas, fue aprobado el Reglamento del Consejo de 1 de febrero de 1983 sobre la apertura de los archivos de la Comunidad Económica Europea y la Comunidad Europea de la Energía Atómica, en virtud del cual se reconoce el acceso del público a dichos archivos tras un periodo de 30 años a contar desde la fecha de producción de los documentos y piezas» (1997, p. 220).

${ }^{11}$ Publicado en el DOCE C-335 de 30-XII-1988, p. 5. 
pública y su difusión, conforme a las disposiciones de la presente Directiva».

El texto derivó en la Directiva 90/313/CEE, de 7 de junio de 1990 que mencionábamos al inicio, sobre libertad de acceso a la información en materia de medio ambiente ${ }^{12}$ que debía entrar en vigor para todos los Estados Miembros antes de 1993. Reflejo de esta indicación es la publicación en España de la Ley 38/1995, de 12 de diciembre de derecho de acceso a la información en medio ambiente y que se demoró en implantarse tres años más de lo permitido.

Esta normativa comunitaria comenzaba así la Exposición de Motivos:

«el acceso a la información sobre medio ambiente que obre en poder de las autoridades públicas mejorará la protección medioambiental...es necesario garantizar que cualquier persona física o jurídica tenga libre acceso en la Comunidad Europea a la información escrita, visual y sonora o de base de datos, que obre en poder de las autoridades (...)».

A continuación, al igual que en la propuesta, el primer precepto señala que «el objeto de la presente directiva es garantizar la libertad de acceso y la difusión de la información sobre medio ambiente que esté en poder de la autoridad pública, así como establecer los plazos y condiciones básicas (...)».

Como normativa vinculante para los Estados Miembros, el art. 3 y 6 de la Directiva 90/313/CEE establecían respectivamente que en el término impuesto se había de implementar todo

«lo necesario para que las autoridades públicas estén obligadas a poner la información relativa al medio ambiente a disposición de cualquier persona física o jurídica que así lo solicite y sin que dicha persona esté obligada a probar un interés determinado» y que efectuasen obligatoriamente «una publicidad activa al respecto».

Casi una década después, de 28 de enero de 2003, la Directiva 2003/4/CE ${ }^{13}$ sobre acceso público a la información medioambiental deroga la inicial Directiva 90/313/CEE, que dotó de mayor alcance al derecho de acceso a la información ambiental a todas las informaciones obrantes al respecto en poder de los Estados y creó un procedimiento de acceso con todos los elementos necesarios (definiciones, plazos, límites, etc.).

A continuación, la Directiva 2003/98/CE ${ }^{14}$ del Parlamento Europeo y del Consejo, relativa a la reutilización de la información del sector público,

\footnotetext{
12 Publicado en el DOCE L-158 de 23-VI-1990, p. 56.

${ }^{13}$ Publicado en el DOUE 41 de 14-II-2003, pp. 26-32.

${ }^{14}$ Publicado en el DOUE 175, de 27-VI-2013, pp. 1- 8.
} 
estableció un conjunto mínimo de normas que regulan actualmente la reutilización y los instrumentos prácticos que facilitan la reutilización de los documentos existentes conservados por organismos del sector público de los Estados Miembros. Esta Directiva entró en vigor en España dos años más tarde de lo indicado ${ }^{15}$ con la Ley 37/2007, de 16 de noviembre, sobre reutilización de la información del sector público.

La normativa dio lugar a que los Estados de la UE reflejen en mayor o menor medida, con mayor o menor premura en sus ordenamientos, premisas de transparencia en sus administraciones.

Mestre (1998, pp. 60 y ss.) defendía ya hace casi veinte años la existencia de la transparencia como un Principio General del Derecho Comunitario basándose en los derroteros de las Directivas, aludiendo a la Declaración 17 relativa al derecho de acceso a la información aneja al Acta Final del Tratado de la UE firmado en Maastricht el 7 de febrero de 1992, según la cual «la Conferencia estima que la transparencia del proceso de decisión refuerza el carácter democrático de las instituciones, así como la confianza del público en la Administración». Así el autor dejaba de manifiesto la fuerte tendencia comunitaria en premisas de calidad democrática, tan importante y evidentes hoy día como lo son abrir los debates sobre programas de trabajo o sobre cuestiones fundamentales de interés comunitario, o la publicación de las actas de votaciones.

\section{III.4. Otras disposiciones comunitarias aledañas}

En otra escala, pero con carácter dispositivo se encuentran en la UE los Códigos de Conducta y el Libro Verde sobre la iniciativa europea en favor de la transparencia. El Libro Verde de 3 de mayo de 2006, es un referente por poner en marcha una consulta pública para mejorar la transparencia del proceso de toma decisiones. Cuando la Comisión lanzó la iniciativa europea en favor de la transparencia, el 9 de noviembre de 2005, distinguió tres ámbitos de acción: (i) el suministro de información más completa sobre la gestión y la utilización de los fondos comunitarios; (ii) la deontología en las instituciones europeas; y, (iii) la regulación de los grupos de presión y las organizaciones civiles. En concreto, instauró una sede virtual para mejorar el control de la utilización de los fondos de la UE que son de gestión compartida, adoptó una serie de medidas que reanudaron el debate sobre las normas deontológicas que deben observar los mandatarios políticos de las instituciones europeas, y revisó la legislación sobre el acceso a los documentos y la revisión del marco jurídico que regula las actividades de la Oficina Europea de Lucha contra el Fraude.

${ }^{15}$ En atención al art. 12 de la Directiva 2003/98/CE que establecía de plazo hasta el 2005. 
Más adelante, a mediados de los años 90, el Consejo de la UE y la Comisión aprobaron el Código de Conducta relativo al acceso del público a los documentos del Consejo ${ }^{16}$ y de la Comisión ${ }^{17}$. El Código presentó como principio general el mayor acceso posible a los documentos que posean la Comisión y el Consejo. Definió además lo que entiende por "documento público": «todo escrito, en cualquier soporte, que contenga datos existentes y que esté en poder del Consejo y de la Comisión». A continuación, reguló también las solicitudes por escrito estableciendo que «deberá contener en particular una serie de elementos que sirvan para identificar el documento o los documentos de que se trate». Si el autor del documento fuese una persona física, jurídica u otro organismo, la solicitud deberá dirigirse directamente al mismo. El acceso a los documentos se realizará, bien mediante consulta in situ, bien mediante copia y la institución podrá disponer que no se realice una difusión del documento o una utilización con fines comerciales.

El Código fue desarrollado por la Decisión 93/731/CEE ${ }^{18}$, de 20 de diciembre de 1993 de acceso del público a los documentos del Consejo y por la Decisión 94/90/CECA, CE, Euratom de 8 de febrero de 1994, a los documentos de la Comisión. Este doble desarrollo consolida el Código con la única novedad en la Comisión de añadir el plazo de un mes para resolver la desestimación de una solicitud y en el Consejo que la ausencia de respuesta determinara la denegación por silencio administrativo negativo, tal como se da en la regulación española.

En el año 2000 la Comisión aprueba el Código de Buena Conducta Administrativa ${ }^{19}$, que destaca los principios fundamentales que deben regir una administración pública europea «centrada en el servicio, la independencia, la responsabilidad, la eficacia y la transparencia», donde los ciudadanos podrán presentar reclamaciones sobre una eventual infracción a los principios codiciales ante la Secretaría General de la Comisión Europea, la misma que las enviará al departamento pertinente. Establece que el Director General o Jefe del departamento contestará al denunciante por escrito en el plazo de dos meses a la reclamación; luego, el denunciante tendrá un mes para solicitar al Secretario General de la Comisión la revisión de la respuesta a la reclamación, a modo de recurso, que será contestada por el Secretario General en el plazo de un mes.

\footnotetext{
${ }^{16}$ Publicado en el DOUE 46 de 18II-1994, pp. 58-61.

${ }^{17}$ Publicado en el DOUE L-340 de 31-XII-1993.

${ }^{18}$ Publicado en el DOUE 325 de 14-XII-1996, p. 19.

${ }^{19}$ Publicado en el DOUE L-267 de 20-X-2000.
} 
El año 2001 el Parlamento Europeo ${ }^{20}$ aprobó el Código Europeo de Buena Conducta Administrativa, donde se introdujeron algunas pautas sobre la regulación del servicio que el ciudadano europeo puede esperar de la Comisión, basadas en unos principios fundamentales de entre los que destacan, el principio de control y publicación de datos estadísticos, el derecho de los ciudadanos a que se les atienda con corrección, y el derecho a la contestación de sus escritos en un plazo reglamentario.

Los principios básicos que contienen estos Códigos de buen hacer administrativo son los que los funcionarios debían observar en su trato con el público. Promueven la adopción de decisiones en un plazo razonable, no superior a dos meses, motivándolas especialmente cuando fueren desfavorables a los derechos de las personas privadas, en cuyo caso debía indicarse los medios de recurso frente a dichas decisiones. Además, los Códigos comunitarios promovían el archivo de los documentos, la publicidad del Código y la reclamación ante el Defensor del Pueblo ante los incumplimientos de la normativa realizados por los funcionarios.

Junto a las estudiadas referencias comunitarias se sugiere la mención de las siguientes medidas políticas y legislativas en materia de transparencia de ámbito europeo, antes de adentrar el foco en la jurisprudencia y los Estados europeos concretos. Dichas disposiciones son:

- Iniciativa europea en favor de la transparencia: el Registro de grupos de interés, un año después de la Comisión de las Comunidades Europeas COM (2009), 28$\mathrm{X}-2009$.

- Decisión de la Alto Representante de la Unión para Asuntos Exteriores y Política de Seguridad de 19-VI-2011, relativa a las normas de acceso a los documentos (2011/C 243/08).

- Informe de la Comisión sobre la aplicación, del Reglamento CE 1049/2001 relativo al acceso del público a los documentos del Parlamento Europeo, del Consejo y de la Comisión Bruselas, 12-VIII-2011 COM (2011) 492 final.

${ }^{20}$ El Parlamento ya había sentado sus intenciones en la materia en la Resolución de 22IV-1994 (Diario Oficial de la UE C-128 de 9-V-1994) sobre transparencia en la UE, en la que sentenciaba que la transparencia

«consiste en el acceso de los ciudadanos a las decisiones de las autoridades de la UE, a sus trabajos preparatorios o relacionados con ellas y, en general, al conjunto de las documentaciones de que disponen dichas autoridades (...) destacando que la transparencia de las distintas instituciones es insuficiente si el conjunto del sistema comunitario permanece opaco para los ciudadanos».

Además, la resolución clarificaba que «los debates y votaciones de las autoridades legislativas deberían ser públicas y, por consiguiente, objeto de una publicación regular rápida, tanto por parte del Consejo, en su función de legislador como por parte del Parlamento Europeo». 
- Reglamento de Parlamento europeo (art. 39).

- Resolución del Parlamento Europeo, de 17-XII-2009, sobre las mejoras necesarias en relación con el marco jurídico de acceso a los documentos a raíz de la entrada en vigor del Tratado de Lisboa (Reglamento CE 1049/2001; 2010/C 286 E/03).

- Comunicación de la Comisión del seguimiento del Libro Verde "Iniciativa europea en favor de la transparencia" COM, 21-III-2007.

- Comunicación de la Comisión: Hacia una cultura reforzada de consulta y diálogo-Principios generales y normas mínimas para la consulta de la Comisión a las partes interesadas COM (2002) 704 final.

\section{III.5. Jurisprudencia europea}

La jurisdicción comunitaria se ha pronunciado sobre el alcance de la transparencia y el derecho de acceso a la información pública en varias ocasiones, normalmente en sentencias de recursos. Las resoluciones más importantes a nivel de contenido jurisprudencial sobre acceso a la información se extraen de demandas entre ONG pro acceso a la información y pro transparencia, contra órganos de la UE y contra Estados miembros respecto a la denegación o permisión de cierta información de carácter público-comunitario.

En primer lugar, se destaca la sentencia del Tribunal de Justicia Europeo T-93/11 de 13 de julio de 2013, la cual tiene por objeto la anulación de la decisión de la Comisión de 6 de diciembre de 2010, denegatoria del acceso íntegro por la demandada a diversos documentos relativos a las negociaciones (actas de grupos de trabajo, posturas de los Estados, identificación de intervinientes, etc.) entre la UE y la República de la India para la firma de un acuerdo de libre comercio. Alemania se une a la causa de parte de la UE en la defensa.

La demandante fue la ONG Stichting Corporate Europe Observatory (Observatorio Corporativo de Europa), con fines de investigación, filantropía política y activista pro acceso. Solicitó más de un centenar de documentos sobre aquellas negociaciones de los cuales la Comisión facilitó una amplia mayoría, pero no todos. El conflicto radica en la denegación a la solicitud del resto, justificada en la aplicación del art. 4.1.a), tercer guion ${ }^{21}$, del Reglamento 1049/2001 del Parlamento Europeo

${ }^{21}$ El Reglamento 1049/2001 dice:

Art. 4.1.a) Excepciones: Las instituciones denegarán el acceso a un documento cuya divulgación suponga un perjuicio para la protección de: a) el interés público, por lo que respecta a:

- la seguridad pública,

- la defensa y los asuntos militares, 
y del Consejo, relativo al acceso del público a los documentos del Parlamento Europeo, del Consejo y de la Comisión ${ }^{22}$.

La ONG alega que no es aplicable dicha norma en la que se escudan «debido a que esos documentos han entrado en el dominio público como consecuencia de las acciones y omisiones de la Comisión» y que ha de corresponderle el trato de agente solicitante como cualquier otro de los permitidos a lo que el propio tribunal detalla:

«(L)a demandante alega que no existe ninguna diferencia destacada entre ella y los sectores empresariales consultados por la Comisión, por lo que ésta actuó de manera discriminatoria al divulgar documentos únicamente a esos sectores. Tanto más es así toda vez que la Comisión no puede demostrar porqué la demandante sería menos fiable o digna de confianza que las organizaciones profesionales destinatarias de los documentos».

Aquí se aprecia la colisión de interpretaciones, junto a un antes y un después en la que las ONG como entes imparciales y sin ánimo de lucro interactuaban vis a vis con las instituciones europeas con la tutela judicial que le corresponde y que le garantizó una amplia mayoría de documentos y el ajustar su estatus al de los agentes económicos con intereses, a pesar de que no se logre la totalidad del petitum.

En esta causa se ponen de manifiesto las controversias entre varios altos principios como el de denegación de documentos confidenciales con el principio de igualdad y no discriminación, donde reiterada jurisprudencia ${ }^{23}$ exige que las situaciones comparables no reciban un trato diferente y que no se traten de manera idéntica situaciones distintas, a no ser que este trato esté objetivamente justificado.

Con este texto judicial se dio un toque mayúsculo de atención a la Comisión para poner fin a su errónea práctica de concesión a las grandes empresas un acceso privilegiado a las políticas comerciales de la UE, a expensas del interés general. Esta discriminación conducía a una política comercial que satisfacía cuidadosamente y con esmero las necesidades de las grandes empresas.

- las relaciones internacionales,

- la política financiera, monetaria o económica de la Comunidad o de un Estado miembro. (...)

${ }^{22}$ Diario Oficial de la Unión Europea L-145 de 30-V-2001, p. 43.

${ }^{23}$ Sentencias del Tribunal de Justicia de 10-I-2006, IATA y ELFAA, C-344/04, Rec. p. I-403, apartado 95, y de 19-VII-2012, Lietuvos geležinkeliai, C-250/11, apartado 44. 
Finalmente, a pesar de desestimar la pretensión del observatorio ${ }^{24}$, se trató de una "media estimación" o una derrota con sabor a gloria, porque se logró más del $80 \%$ de los documentos solicitados, logró vencer por vía extrajudicial algunos de los límites inicialmente impuestos y dar visibilidad a una situación de desigualdad en el acceso a la información, entre otros resultados positivos.

Otra sentencia a destacar es la C-280/11 P de la Sala Primera del Tribunal de Justicia de la Unión Europea de 17 de octubre de 2013 en casación $^{25}$, Acces Info Europa vs. Consejo de la UE, en la que se rechazan los argumentos formulados por el Consejo y por el gobierno de nuestro país que pretendían la reserva la identidad de los Estados Miembro a la hora de proponer enmiendas legislativas durante los debates en el Consejo, así como en cuestiones de posicionamiento de intereses.

En un principio, el Consejo defendió su férrea política de publicar documentos sobre un proceso legislativo cuando estuvieren tachados los nombres de los Estados miembros, garantizando una protección de identidad comercial ${ }^{26}$ para que no se pudiera saber quién se encuentra detrás de las enmiendas y proposiciones presentadas. Conjuntamente, el Gobierno español esgrimió que era mejor mantener el anonimato sobre quiénes intervenían en cada enmienda para «evitar posibles presiones» sociales en cada Estado, lo cual representa un incordio en la acción política esquivando controles ciudadanos.

Las acciones de la ONG fueron estimadas ante el Tribunal General en marzo 2011, el cual falló que la ONG tenía derecho de acceso a la información a un documento que solicitó en 2008; sin embargo, el Consejo recurrió la decisión, con el apoyo de los gobiernos de España, Francia, Grecia, el Reino Unido y la República Checa. El Parlamento Europeo, que argumentaba a favor de una mayor transparencia del proceso legislativo, se unió al caso, dando un paso histórico al apoyar a la ONG y su misión de aumentar el acceso público a documentos europeos.

El Tribunal Europeo de Justicia rechazó los argumentos del Consejo que buscaban legitimar la no publicación de la información completa para «proteger la eficacia del proceso de toma de decisiones», a lo que el

\footnotetext{
${ }^{24}$ El Tribunal recordó que el interés público no se puede tomar en consideración en el marco de la aplicación de las excepciones obligatorias previstas en el art. 4.1, a), del Reglamento 1049/2001 (sentencia Sison/Consejo, apartado 52 supra, apartado 52, confirmada por sentencia del Tribunal de Justicia de 1-III-2007, Sison/Consejo, C-266/05 P, Rec. p. I-1233, apartado 47), como señaló la Comisión en la Decisión impugnada.

${ }^{25}$ La primera resolución del Tribunal General de la UE es la T-233/09 de 22-III-2011.

${ }^{26}$ Se sugiere la lectura de los estudios referentes al secreto comercial o industrial desde la óptica española contenidas en la obra de Mestre, 1998, pp. 176 y ss.
} 
Tribunal sentenció que «la mera circunstancia de que la solicitud de divulgación se presentara en una fase muy temprana del procedimiento legislativo no era suficiente para permitir la aplicación de esa excepción», además de que «el gobierno Español debería integrar estos estándares de transparencia en todo el proceso legislativo, algo que no se contempla en la ley de transparencia, a punto de ser aprobada».

Como se ve, los ciudadanos españoles tendrán la legitimación europea a la hora de acceder a información europea, de tener un parlamento más transparente y de saber qué es lo que ocurre en Bruselas, lo cual hará más fácil a los eurodiputados el escrutinio de los procesos legislativos al conocer cuál es la posición que adopta cada país durante las negociaciones. Además, con esta resolución, como con muchas otras y gracias a la iniciativa de entidades como las ONG, se refuerza la legitimación de los ciudadanos ante las leyes y directivas europeas que nos afectan, aumentando además el control del eurociudadano sobre sus instituciones y Estados correspondientes para asegurar una rendición de cuentas del poder legislativo estatal y del de la Unión Europea.

Una de las posturas jurisprudenciales más antiguas de la UE a favor del derecho de acceso es la Sentencia del Tribunal de la UE Asunto T194/94 de 19 de octubre de 1995 de John Carvel y Guardian Newspapers contra el Consejo (cfr. Mestre, 1998, pp. 67-71). En esta causa también una institución comunitaria deniega el acceso a documentos, con las mismas intenciones que las antes indicadas: proteger intereses particulares y proteger de la opinión pública ciertas decisiones. En este caso se protegían las posturas de los Estados frente a asuntos de Justicia, Agricultura y Asuntos Sociales europeos sosteniendo que «era preciso cuidar el secreto de las deliberaciones ya que los documentos de que se trataba contenían informaciones confidenciales relativas a la postura adoptada por los miembros del Consejo durante sus deliberaciones (...)».

El Tribunal no dudo y respondió a tales argumentos estimando la petición de la parte demandante y subrayando - como recuerda Mestreque se ha de ponderar tanto el interés del ciudadano, como el interés público del ciudadano en la obtención de documentos. Por el secreto de las deliberaciones no se puede privar a los ciudadanos del acceso, absteniéndose de la facultad del art. 5.1 de Reglamento interno (derivados del art. 4.2 de la Decisión 93/731 relativa al acceso del público a los documentos del Consejo).

Esta es, en resumidas cuentas, la doctrina europea que en este aspecto apunta, no en balde, a la protección del derecho de acceso y a tutelar su garantía frente a las armaduras institucional-comunitarias que se venían practicando. 


\section{III.6. Art. 255 del Tratado de Ámsterdam}

Aludiendo ahora a un precepto de interés del Tratado de Ámsterdam y una vez repasado el perfil europeo respecto a la transparencia, especialmente el de las autoridades judiciales, ha de hacerse un comentario a una punta de lanza en el acceso a la información en el ámbito comunitario, el art. 255 del Tratado (vigente desde el 12 de junio de 1985 hasta el 1 de diciembre de 2009), que dice:

Art. 255.1. Todo ciudadano de la Unión, así como toda persona física o jurídica que resida o tenga su domicilio social en un Estado miembro, tendrá derecho a acceder a los documentos del Parlamento Europeo, del Consejo y de la Comisión, con arreglo a los principios y las condiciones que se establecerán de conformidad con los apartados 2 y 3 .

2. El Consejo, con arreglo al procedimiento previsto en el artículo 251, determinará los principios generales y los límites, por motivos de interés público o privado, que regulan el ejercicio de este derecho de acceso a los documentos, en el plazo de dos años a partir de la entrada en vigor del Tratado de Ámsterdam.

3. Cada una de las instituciones mencionadas elaborará en su reglamento interno disposiciones específicas sobre el acceso a sus documentos.

Este precepto, de 23 años de historia en los años preadolescentes de la UE, se pronunció sobre la transparencia y el acceso a la información en un momento en el que se modernizó y modificó el Tratado de la Unión Europea, donde se constituyó una definición completa de la UE institucionalmente. Esta norma puede decirse, en palabras de Mestre (1998, p. 73), que fue la que consolidó el principio de transparencia comunitario.

La doctrina académica inmediatamente posterior a la aprobación del Tratado de Ámsterdam discrepaba sobre la tesitura, alcance e importancia de este artículo. Sin embargo, autores como Guichot (2011, pp. 296-297) le asignaron un rango totalmente fundamental y necesario basándose en los estudios de derecho, tanto legislativos como judiciales, que habían puesto de manifiesto que este era un derecho desde mucho antes de que se tratase de reconociera en todos los Estados miembros, como ocurre a día de hoy. A su juicio del mismo Guichot (2011, pp. 82-85), el juez comunitario ya había reconocido que la normativa, como derivación del principio democrático, era inmanente al funcionamiento de las instituciones, aunque pudiera ser objeto de regulación administrativa.

Es destacable en primer término que la regulación sobre acceso a documentos administrativos del art. 255 tenía un carácter aperturista, conciliador e inclusivo. Puede parecer que existió una fuerte influencia escandinava y de los Países Bajos, que dedicaron a la transparencia un espacio propio en un artículo separado, aunque no de manera principal. 
Podría ser esta norma el precedente de los Códigos de Conducta que vinieron luego y que se han manejado en la presente obra.

A partir de la norma en cuestión la interpretación de las Decisiones aprobadas bajo el Código de Conducta de 1993 hizo recaer una ardua labor de armonización jurídica en los entes jurisdiccionales comunitarios, pues ahora existía un cimiento constitucional en los Tratados, aunque no se definía taxativamente el alcance máximo que poseía.

Este alcance inexorablemente estaba sujeto a la categoría que se concediera al derecho en el seno del sistema jurídico europeo. Así pues ha de decirse que tras la aprobación del art. 255 la jurisprudencia resultó heterogénea y a veces errada, poniendo de manifiesto que en el momento de la publicación de la norma estaba en proceso de redacción el esencial Reglamento 1049/2001 relativo al acceso del público a los documentos del Parlamento Europeo, del Consejo y de la Comisión.

Puede evidenciarse la importancia de esta norma de transparencia de Estado comunitario en una resolución internacional, como la que el Tribunal de Justicia Europeo ${ }^{27}$ dictó cuando estimó el acceso a un informe de un fondo dedicado al comercio de armamento que aprobó el mismísimo Comité Político del Consejo Europeo y que contenía los criterios de coordinación al respecto de los Estados implicados.

En la resolución se interpretó la Decisión $93 / 731 / \mathrm{CE}^{28}$, relativa al acceso del público a los documentos del Consejo y se llegó a la conclusión de que «el régimen de excepciones de la Decisión debía ser vista a la luz del principio del derecho de acceso a la información para lograr su efectivo ejercicio por parte de la ciudadanía». Se crea así el criterio de que cuando dentro de un mismo documento público cierta información no se encuentra justificada por alguna de las excepciones tasadas, la misma ha de ser puesta de manifiesto en virtud del principio de proporcionalidad. En suma puede considerarse con esto que el Código de Conducta de 1993 y las Decisiones institucionales que lo complementan, reconocen el derecho de acceso a la información. Guichot (2011, pp. 61-62) señala que los documentos no pueden considerarse como un todo indivisible a la hora de su entrega:

«Si tan sólo una parte de su contenido está cubierta por alguna de las excepciones, debe facilitarse el acceso al resto. Contrario a esta apreciación de los países con sistemas informativos más abiertos, el Consejo, con el significativo apoyo de España y Francia, defendió la posición contraria» (2011, p. 106).

\footnotetext{
${ }^{27}$ Sentencia del Tribunal de Primera Instancia de 19-VII-1999, Asunto 14/98.

${ }^{28}$ Diario Oficial de la UE 325 de 14-XII-1996, p. 19.
} 
Durante la ratificación del art. 255 y la adopción del Reglamento del año 2001, mucha de la discusión se centró en el alcance y contenido de las limitaciones vigentes, pues a nadie pasaba desapercibido que estas serían la clave y el motor del futuro sistema de acceso público a los documentos comunitarios. Tampoco resultaba ser un secreto que la interpretación que de las mismas se hiciera por parte de la jurisdicción comunitaria durante esta laguna temporal jurídica tenía alta probabilidad de trasladarse a la reglamentación. Así que era el momento perfecto para intentar corregir el sistema de sus males originales.

Es de justicia apuntar que todavía en ese periodo las instituciones comunitarias denegaron documentos a solicitantes alegando no ser la que originó primero el documento en su poder, cuando en opinión del que escribe el acceso a un documento no puede simplemente denegarse señalando que pertenece a determinada categoría sin más. La respuesta al solicitante debe expresar de forma clara e inequívoca, los criterios y los fundamentos de la denegación, pues de lo contrario éste no podría formular argumentos en defensa de sus derechos.

Mientras tanto se encontró al Consejo incurso en una infracción a la Decisión 93/731. No supo explicar por qué el acceso a los documentos solicitados ponía en peligro las relaciones internacionales de la UE.

Otra resolución posterior del Tribunal de Justicia $\mathrm{UE}^{29}$ ratificó otro elemento esencial para el estudio de la permisión institucional de las excepciones al acceso, con dos vertientes del proceso analítico que se debe utilizar al valorar cuándo se usa como pretexto la protección de los intereses de terceros para denegar frívolamente un documento. Las excepciones de pretexto que la institución puede alegar a su discreción pueden ser: (i) las obligatorias que recogen el deber de proteger los intereses de terceros o el interés del público, en general amparado en términos facultativos; y, (ii) puede ampararse en la protección de deliberaciones internas para mantener intactas las relaciones comunitarias con los Estados.

Estas excepciones pueden coincidir, aunque el escrutinio de cada una se hará por separado. El Tribunal llegó a la conclusión de que no se había

\footnotetext{
${ }^{29}$ Caso Denkavit Nederland BV vs. Comisión del STPI de 13-IX-2000, Asunto T-20/99 (Rec. 2000, p. II-3011) en el que el demandante solicitó a la Comisión acceso a un informe de inspección de 1997 sobre la lucha en los Países Bajos contra la fiebre porcina. El documento incluía los resultados de una investigación sobre los gastos hechos en el control de los fondos de emergencia. Con arreglo al plan aprobado, Holanda obtuvo un primer anticipo de 31.3 millones de euros destinados a indemnizar a los propietarios de las explotaciones afectadas (cantidad pagadera previa a la presentación de justificativos). Se denegó el documento por suponer un perjuicio a la protección de los intereses públicos.
} 
cometido ningún error de apreciación al estimar que la divulgación del informe solicitado podía suponer un perjuicio para la protección del interés público, estimando innecesario entrar a considerar si la denegación del informe estaba justificada igualmente por la protección de los intereses de terceros en su modalidad dedicada al resguardo del secreto comercial.

Finalmente, el art. 255 tiene los límites señalados en el apartado 2. No son ilimitadas «las declaraciones específicas como la posibilidad que asiste a los Estados miembros de solicitar a la Comisión o al Consejo que no se comunique a terceros un documento originario de dicho Estado sin su consentimiento (...)» (Mestre, 1998, p. 74).

\section{CONCLUSIONES}

1. Desde 1766 existe un expansivo interés y una preocupación pública internacional en desarrollar normativas de transparencia y acceso a la información pública en cada uno de los poderes del Estado.

2. Cada Estado otorga una prioridad a la transparencia que suele coincidir con la calidad de su ordenamiento jurídico. A veces se enmarca el derecho de acceso en una categoría fundamentalista o afundamentalista, ignorando las premisas internacionales a los que está sometido.

3. Las organizaciones internacionales han previsto el alcance de la información pública en sus declaraciones y otras manifestaciones, de las que se han inspirado los legisladores de muchos Estados del mundo.

4. Muchos países latinoamericanos ya poseían un rico desarrollo en la materia antes que comenzase la reciente tendencia de transparencia y acceso como respuesta a las prácticas deshonestas en la cosa pública y les dedicaban en sus ordenamientos una considerable atención y espacio.

5. La UE, por medio de sus cauces oficiales, se ha ocupado de transmitir a los Estados miembros las máximas categóricas de la transparencia y el derecho de acceso a la información pública, definiendo un carácter común supranacional comprometido con estos valores.

6. Existe una gradual diferencia de España con las normativas coetáneas y colindantes respecto al alcance que concedido a sus normas. La reciente normativa española aún es reticente en considerar el derecho de acceso como derecho fundamental, a pesar del potente aparato legal e institucional que ha tenido en otras normas.

7. España ha recibido un impulso considerable en la materia gracias a que forma parte de la Unión Europea. Se ha nutrido de la normativa comunitaria que al respecto de la transparencia se han pronunciado en numerosas ocasiones aquí analizadas. 


\section{REFERENCIAS}

Álvarez Vélez, M. y Alcón M. (1996). Las constituciones de los quince Estados de la Unión Europea. Madrid: Dykinson.

Castañeda, J. (1967). Valor jurídico de las resoluciones de las Naciones Unidas. Ciudad de México: Colegio de México.

Cotino Hueso, L. (2005). El nuevo derecho fundamental europeo al acceso a los documentos, transparencia e información pública. Libro en Homenaje a Íñigo Cavero Lataillade. Valencia: Tirant lo Blanch, pp. 725-753.

Diez de Velasco, M. (2009). Instituciones de derecho internacional público. Madrid: Tecnos.

Fernández Ramos, S. (1997). El derecho de acceso a los documentos administrativos. Madrid: Marcial Pons.

Guichot, E. (2011). Transparencia y Acceso a la Información en el Derecho europeo. En Cuadernos Universitarios de Derecho Administrativo. Sevilla: Derecho Global.

Guichot, E. (2014). Transparencia y acceso a la información pública en España: análisis y propuestas legislativas. Documentos de trabajo (Laboratorio de alternativas), $170,2011$.

Mendel, T. (2008). Libertad de información: Comparación jurídica. París: UNESCO.

Mestre Delgado, J. (1998). El derecho de acceso a los archivos y registros administrativos. Análisis del artículo 105.b) de la Constitución. Madrid: Civitas.

Quirós Soro. F. (2012). Transparencia en la Unión Europea. Métodos de información, 5(3), pp. 177-203.

\section{Normativa:}

Carta de los Derechos Fundamentales de la Unión Europea, del Parlamento Europeo, el Consejo de la UE y la Comisión Europea firmada el 7-XII-2000 en Niza.

Carta Democrática Interamericana, aprobada el 11-IX-2001.

Carta Iberoamericana de Participación Ciudadana en la Gestión Pública, adoptada en la XIX Cumbre Iberoamericana, Portugal, 2009.

Código Europeo de Buena Conducta Administrativa, del Parlamento Europeo, 2001.

Constitución Española, 1978.

Convención Americana sobre Derechos Humanos, 1969.

Convenio del Consejo de Europa sobre el Acceso a los Documentos Públicos, firmado en Troms (Noruega), el 18-VI-2009.

Convenio Europeo para la Protección de los Derechos Humanos y de las Libertades Fundamentales, adoptado por el Consejo de Europa el 4-XI-1950. 
Convenio sobre acceso a la información, participación del público en la toma de decisiones y acceso a la justicia en materia medioambiental, 1998.

Decisión 94/90/CECA, sobre acceso público a documentos de la Comisión Europea.

Decisión del Consejo por la que se hacen accesibles al público determinadas categorías de documentos del Consejo, 9-IV-2001.

Declaración Universal de los Derechos Humanos, 10-XII-1948.

Directiva 2003/4/CE del Parlamento Europeo y del Consejo relativa al acceso del público a la información medioambiental y por la que se deroga la Directiva 90/313/CEE del Consejo, 28-I-2003.

Directiva 2003/98/CE del Parlamento Europeo y del Consejo, relativa a la reutilización de la información del sector público, 17-XI-2003.

Directiva 90/313/CEE, del Consejo, de 7-VI-1990, sobre libertad de acceso a la información en materia de medio ambiente.

España, Ley 19/2013 de 9-XII-2013 de Transparencia, acceso a la información pública y buen gobierno.

España, Ley 30/1992 de 26-XI-1992, de Régimen Jurídico de las Administraciones Públicas y del Procedimiento Administrativo Común.

España, Ley 37/2007 de 16-XI-2007, sobre reutilización de la información del sector público.

España, Ley 38/1995 de 12-XII-1995 sobre el Derecho de Acceso a la Información en materia de Medio Ambiente.

Iniciativa europea en favor de la transparencia: Libro Verde de 3-V-2006.

Instrumento de ratificación del Convenio de lucha contra la corrupción de agentes públicos extranjeros en las transacciones comerciales internacionales, París, 17XII-1997.

Ley Modelo Interamericana de Acceso a la Información Pública, 8-VI-2010.

Pacto Internacional de Derechos Civiles y Políticos, aprobada mediante la Resolución 2200 A (XXI), de 16-XII-1966.

Suecia, Ley de Libertad de Prensa, 1766.

Tratado de Ámsterdam, 2-X-1997.

Tratado de la Unión Europea, 7-II-1992.

Tratado de Lisboa, 13-XII-2007. 